\title{
EVALUATION OF USE OF PLATELET RICH FIBRIN COMBINED WITH AUTOGENOUS ILIAC BONE GRAFT FOR CLOSURE OF UNILATERAL ALVEOLAR CLEFT
}

\author{
Ahmed S. Naguib*
}

\begin{abstract}
Purpose: The aim of this study was to evaluate the use of platelet rich fibrin combined with autogenous iliac bone graft for closure of unilateral alveolar cleft.

Patients and Methods: Eight patients with unilateral alveolar cleft were included in this study, 5 patients were males and 3 patients were females, their ages ranged from 9-11 years with a mean of 8.9 years. All patients were evaluated preoperatively both clinically and radiographically. All clinical and radiographic parameters were performed immediate, 1, 3and 6 months postoperatively.

Results: All patients showed uneventful wound healing, without any evidence of occurence of wound infection except in case no. (7), who showed presence of infection after one month but the case was improved through the late follow-up with administration of I.V. antibiotics. Regarding to extrusion of iliac crest graft, no graft extrusion occurred through all the follow-up periods. The visual analogue pain scale (VAS) revealed no pain after 6 months follow-up periods in all patients. All patients showed uneventful closure of the alveolar arch and no oronasal fistula had been detected in any patients through all the follow-up periods. Radiographic evaluation revealed proper height and density of the inserted iliac crest graft.
\end{abstract}

Conclusion: The use of combined mixture of platelet rich fibrin (PRF) and autogenous chips of iliac crest graft is safe and efficient method for performing repair to the alveolar cleft as this mixture leads to occurence of proper increase in bone height and density of the repaired alveolar cleft.

\section{INTRODUCTION}

The alveolus is considered as a part of the primary palate. Nose, Prolabium, lip and premaxilla also arises from the primary palate but all these structures lies in front of the incisive foramen. ${ }^{(1)}$
The alveolus is formed by fusion of the maxillary prominences which occurs at $5^{\text {th }}$ to $6^{\text {th }}$ weeks of gestation. Failure of occurrence of this fusion of the maxillary prominences leads to occurrence of alveolar cleft. ${ }^{(1)}$

* Ass. Professor, Oral \& Maxillofacial Surgery Dept., Faculty of Dentistry, Tanta University. 
The alveolar cleft initially was not considered as a problem on the same order as the cleft lip, and no alveolar repair attempt had been known till (1901), when the Von Eiselberg used the $5^{\text {th }}$ digit for closure of the alveolar cleft. ${ }^{(2)}$

The main objectives for performing repair to the alveolar cleft includes: close the oronasal fistula , maintain the continuity of the maxillary arch, restore the symmetry of the nose, allow occurrence of eruption of the permanent teeth in the newly formed bone, improve speech and phonetics, improve oral hygiene and finally to facilitate orthodontic movement and placement of dental implants. ${ }^{(3)}$

Reconstruction of the maxillary alveolar cleft is very important in order to restore jaw function and morphology. So, use of alveolar bone grafting is considered one of the golden solutions for closure of the alveolar cleft depending upon the osteogenic potential in the osseous defects. ${ }^{(4)}$

The use of Fresh autogenous bone graft is an ideal graft for performing alveolar bone grafting, as it supplies a living immuno compatible osteogenic cells which are necessary for osteogenesis. ${ }^{(5)}$

Autogenous bone graft can be obtained from iliac crest, mandible, tibia or calvarium. However, it had been reported that, autogenous iliac crest bone graft offers a better results, when compared with other alveolar grafts. ${ }^{(6)}$

However, the main draw back for utilizing autogenous bone grafts for reconstruction of the alveolar cleft defects includes donor site morbidity and limited amount can be obtained. So, to avoid these draw backs utilizing of a mixture of autogenous bone graft combined with platelet rich fibrin offer a marvelous results. ${ }^{(7)}$

Platelet rich fibrin (PRF), which is considered as the second generation concentrate, is considered to be superior than the traditional first generation platelet rich plasma (PRP) as it have the following advantages, no handling with the blood and it can be prepared easily. ${ }^{(7)}$

Many studies reported that utilizing the combined mixture of autogenous bone substitute with platelet rich fibrin (PRF) have the following advantages: improve wound repair, enhance bone growth, allow occurrence of excellent wound sealing and facilitate handling of the bone grafts as (PRF) is a gel-like material, and it can be used for preforming a guided bone generation. Also, (PRF) is rich in growth factors that can improve both bone quantity and bone quality. ${ }^{(8)}$

The purpose of this prospective study was, to evaluate the use of platelet rich fibrin (PRF) combined with autogenous anterior iliac crest bone graft for closure of unilateral alveolar cleft both clinically and radiographically.

\section{MATERIALS AND METHODS}

Eight patients with unilateral alveolar cleft were included in this study. Five patients were males and three were females. All patients were selected from the outpatient clinic of Oral and Maxillofacial Surgery Dept., Faculty of Dentistry, Tanta University. The patient's age ranged between 9 to 11 years. All the included patients, their parents or relatives signed an informed consent to participate in this study.

\section{Inclusion criteria}

The patients that were included in this study were:

1. Had good oral hygiene.

2. No history of jaw irradiation.

3. Free of any systemic diseases, especially the bleeding disorders. 
4. Free from any local pathology in the upper jaw, that may interfere with surgery.

5. Had not performed any previous attempt to repair the present alveolar cleft.

6. Absence of any other oronasal fistula

\section{Patient assessment:}

A comprehensive preoperative evaluation of all patients (including patient history, clinical examination (fig. I) and radiographic examination) was performed to determine the main chief compliant, presence of erupting teeth in the cleft and finally to determine the shape and volume of the alveolar cleft defect.

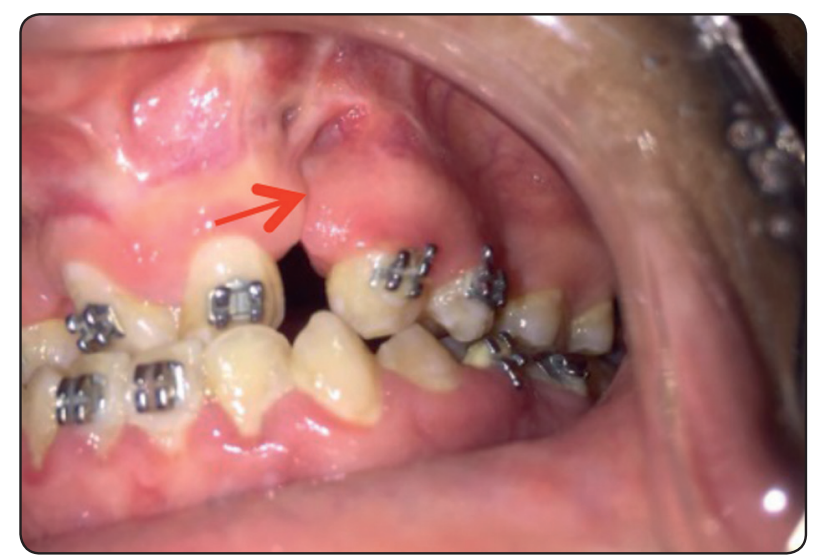

Fig. (I): Preoperative image showing presence of left alveolar cleft (case no. 3).

Preoperative coronal, axial and 3D computed tomography (CT) scans (fig. II) were performed for each patient to assess the morphology of the cleft defect, presence of erupting teeth or supernumerary teeth in the cleft defect, development of the root length, stage of eruption of the permanent lateral incisor and canine. The axial cuts were $0.5 \mathrm{~mm}$ thick and the obtained data from the axial cuts of the CT scan were used to assess and measure the local bone mineral density preoperatively and 1,3 and 6 months after surgery to determine the amount of bone fill of the grafted material.

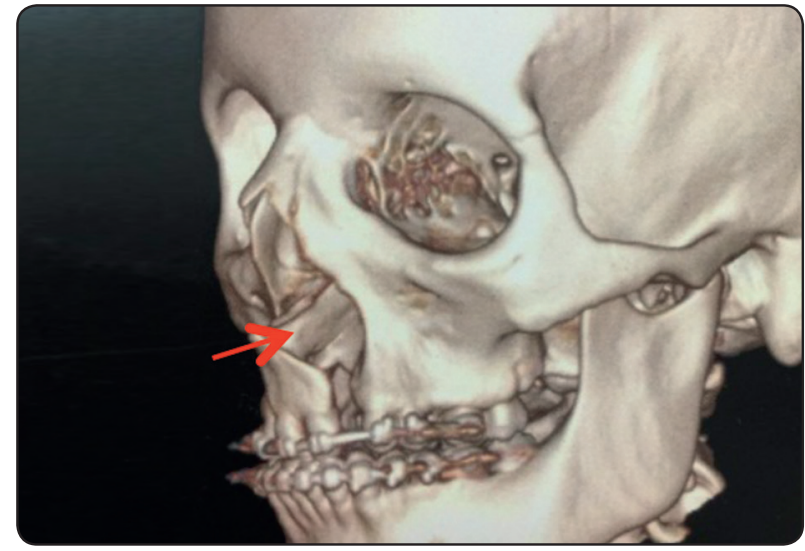

Fig. (II): Preoperative 3D CT scan showing presence of left alveolar cleft (case no. 4).

\section{Surgical technique:}

All patients were operated under general anaesthesia with nasoendotracheal intubation through the noncleft side. The patient was adjusted in supine position. This allows simultaneous preparation of the donor site for harvesting graft from the anterior aspect of the iliac crest. A sand bag was placed under the ipsilateral buttock and standard site preparation was performed.

The surgical field was scrubbed using concentrated Betadiene (Povidene iodine 1\%) and draped with surgical towels. A local anaesthetic solution (Mepivacaine $\mathrm{Hcl} 2 \%$ with levonordefrin $1 / 20000$ ) was injected along the intraoral operative site for performing haemostasis and to facilitate separation of tissue.

\section{Steps of surgical technique:}

\section{Separation of the oral layer from nasal layer}

- Full thickness buccal gingival flaps were performed in the cleft side.

- The nasal mucosa was separated from the oral mucosa by blunt dissection and the alveolar bone was exposed bilaterally.

- The nasal mucosa was then reflected and sutured (water tight nasal closure) 


\section{Preparation of the autogenous anterior iliac crest graft}

A marking pen was used to mark the iliac crest and the anterior superior iliac spine (ASIS) was palpated. the incision area was infiltrated with Mepevacaine 2\% local anaesthesia wit 1/20000 Levonordefrin. The incision was placed lateral to the iliac crest by displacing the skin medially by the nonscalped bearing hand. The incision was done through the displaced skin over the iliac crest, along the bikini line. The limits of the incision not extending to within $1 \mathrm{~cm}$ of the tubercle posteriorly to the anterior superior iliac spine. (fig. III)

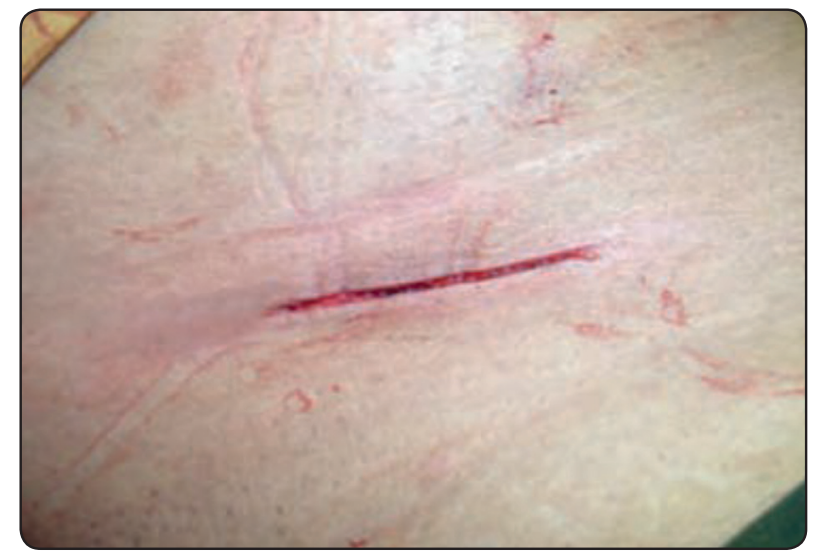

Fig. (III): Intraoperative image showing the extent of the anterior iliac crest incision.

Then perform blunt dissection through the subcutaneous tissue till reaching to the insertion of oblique fascia of the crest. Then incise the fascia and the underlying periosteum to expose the iliac crest. The bone was harvested between the posterior iliac tubercle and the (ASIS) as here, there is greatest width of bone, so facilitating obtaining abundant amounts of cancellous bone.

Harvesting of bone can be achieved by anterior and posterior stop cuts which are joined with a crestal sagittal cut with a small osteotome. The cancellous bone graft was harvested from the medial aspect of iliac crest using curettes.
So, the corticocancellous block of bone and the cancellous bone were obtained. (fig. IV) Before closure, through irrigation was done and meticulous hemostasis was obtained and then a two layer closure was performed to approximate fascia and the subcutaneous tissues and final layer of continuous suture was used to suture the skin.

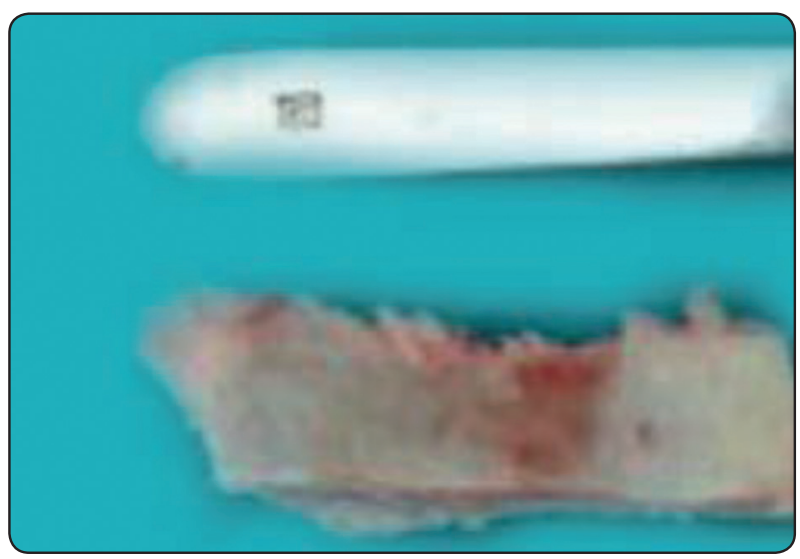

Fig. (IV): Intraoperative image showing the corticocancellous anterior iliac crest bone graft block.

\section{Preparation of the platelet rich fibrin $(P R F)$}

$20 \mathrm{ml}$ of fresh venous blood was drawn from the patient and then each $10 \mathrm{ml}$ of blood was placed in clean glass tubes and shaken well to avoid occurrence of blood clotting. The two tubes were placed into a centrifuge which was adjusted to 3000 rpm for 10 minutes, according to steps of Dohan et al., 2006. ${ }^{(9)}$

After that, the tubes were removed from the centrifuge, which are formed from three layers: In the upper zone platelet poor plasma, in the middle zone a platelet rich fibrin (PRF) and in the lowest zone red blood cells. Then a scissor was used to separate the platelet rich fibrin (PRF) to be prepared for use. (fig. V)

\section{Insertion of the iliac graft and (PRF) in the cleft defect}

The PRF membrane was inserted over the nasal layer. Then, the palatine bone and base of 
the nose were filled with cancellous bone graft and the corticocancellous block bone graft was then contoured on this cancellous bone and inserted with resistance within the cleft area. (fig. VI) Then $2^{\text {nd }}$ PRF membrane was applied over the bone graft. Then the gingival mucoperiosteal flaps were shifted to cover the cleft area and then the oral flaps were sutured primarily.

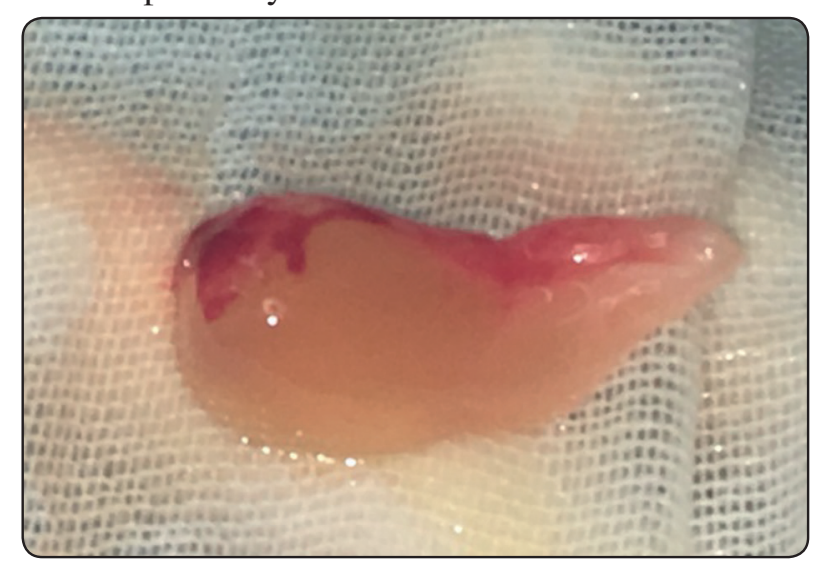

Fig. (V): Intraoperative image showing the platelet rich fibrin membrane.

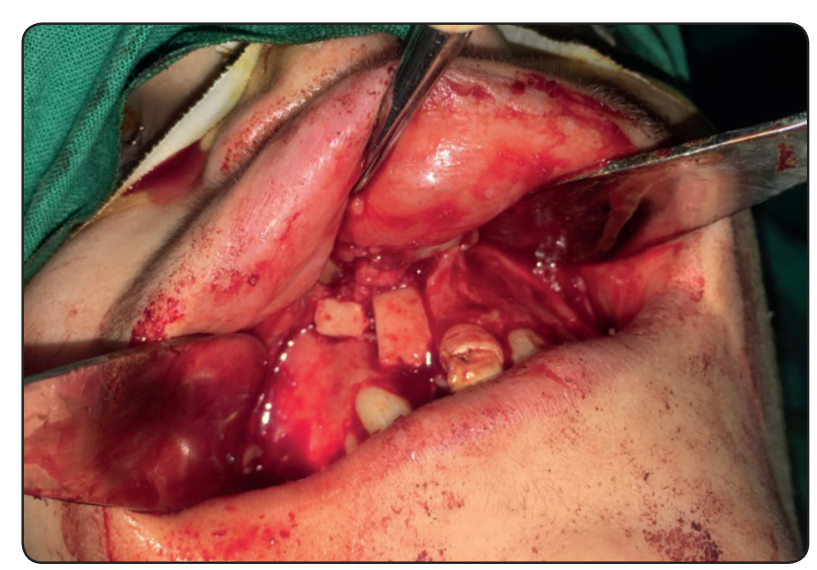

Fig. (VI): Intraoral image of unilateral alveolar cleft at right side of maxilla (case no. 8) was filled with the corticocancellous bone graft and PRF membrane was applied beneath it.

\section{Postoperative care}

Patients were encouraged to take soft diet for 1 week and asked to avoid performing sucking and asked to maintain good oral hygiene with
Betadine mouth wash and performing brushing to the remaining teeth. All patients were given proper antibiotic (Cephalosporin 1gram every 12 hours for 1 week), pain reliefing medication (Voltaren $75 \mathrm{mg}$ amoule every 12 hours for one week) and initial dose of corticosteroids immediately after surgery to reduce postoperative odema (one vial of Ibidron).

\section{Postoperative follow up}

All patients were followed up postoperatively both clinically and radiographically at the following periods [immediate postoperative, one month, 3 months and 6 months].

\section{A) Clinically:}

All patients were followed up to evaluate the following:

1. Wound healing.

2. Presence of infection.

3. Occurrence of extrusion of the graft.

4. Presence of pain (according to visual analogue scale) in the repaired alveolar cleft.

5. Presence of oronasal fistula, with any evidence of nasal regurgitation.

\section{B) Radiographically:}

The coronal CT views were used for determination of the graft height postoperatively (through the follow up periods i.e. immediate, postoperative, 1 month, 3 months and 6 months). The area of the cleft was traced on each coronal cut using mimics software and then the bone height was calculated on each cut starting from the alveolar bone crest up to the anterior nasal spine. Axial CT scans were performed at the same follow up periods to measure the local bone mineral density at each follow up period. 


\section{RESULTS}

\section{A) Clinical results}

There was no severe clinical complications in all surgically treated patients, as shown from the following parameters throughout the follow up periods. (fig. VII)

\section{Wound healing}

All cases showed uneventable wound healing during the postoperative follow up periods.

\section{Presence of infection}

All patients healed properly, as shown through the follow-up periods. Only one patient (case no. 7) showed presence of superficial infection at the wound site after one month from operation, but this patient was improved by intra venous injection of antibiotics (1gm Cefotrixime/12 hours for 5 days) and the patient was improved through the follow up periods (i.e. after 3 months and 6 months).

\section{Occurrence of extrusion of the iliac crest graft:}

Through the different follow up periods there were no instances of migration nor extrusion of the iliac graft had occurred in any case.

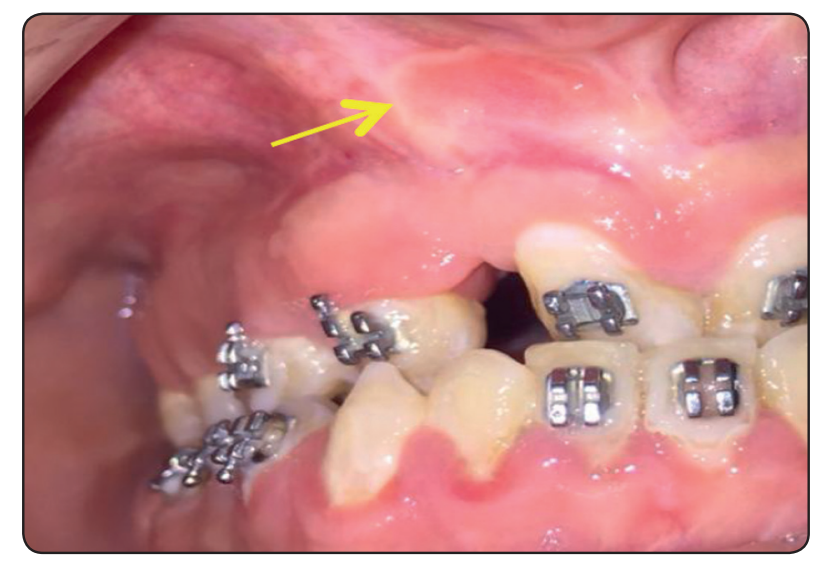

Fig. (VII): Postoperative image showing occurance of proper postoperative healing in the repaired right alveolar cleft. (case no. 5).

\section{Presence of pain (according to visual analogue scale (VAS)) in the repaired alveolar cleft:}

According to (VAS) results, all cases showed mild pain immediately after surgery and after one month from surgery, but no pain had been detected at the third and six months postoperatively.

\section{Presence of oronasal fistula, with any evidence of nasal regurgitation:}

All patients gained uneventful closure of the alveolar arch and no oronasal fistula had been detected in any case nor any nasal discharge had been seen through the follow up periods.

\section{B) Radiographic results:}

All patients exhibited uneventful postoperative course, as shown from the following parameters throughout the follow-up periods:-

\section{Determination of the iliac graft height (through follow-up periods):}

Coronal CT views showed occurrence of proper graft height postoperatively. As the mean bone height of the inserted bone height of the inserted bone graft was $64.61 \mathrm{~mm}$ immediately postoperative and this value decreased with time (i.e. after 1, 3 months respectively) to $47.12 \mathrm{~mm}$ and $49.36 \mathrm{~mm}$ and finally the mean of height of the inserted graft after 6 months was $78.22 \mathrm{~mm}$.

\section{Measuring the bone mineral density in the re- paired cleft:}

The results of serial axial CT scans showed proper bone density in the grafted region of the alveolar cleft over time (through the follow up periods), as the mean of bone mineral density of bone graft was $457 \pm 31.3$ immediately postoperative and this value decreased with time (i.e. after 1 month) to $420 \pm 18.3$ and then increased to $460 \pm 29.2$ time (i.e. after 3 months) and finally increased to $485 \pm 32.1$ time (i.e. after 6 months). 


\section{DISCUSSION}

Today, for management of the alveolar cleft, alveolar bone grafting is considered as an ideal treatment. As grafting of the alveolar cleft stabilize the alveolar arch of maxilla and induce teeth eruption. This result agrees with Hartel et. al., (1999), who showed grafting in the alveolar cleft, gives these same results. In addition, dental implants can be inserted in patients with congenitally missed teeth. ${ }^{(10)}$

The results of this study showed that, the iliac crest is preferable grafting material, for performing repair to the alveolar cleft, as it can be harvested easily and offers sufficient amounts of both cancellous and cortical bone. This result agrees with Dempf et al., (2002), who showed that, the iliac cancellous bone is the preferable grafting material. ${ }^{(11)}$

We aimed to utilize (PRF) with iliac crest graft to enhance the osteogenesis of the autogenous bone graft and the result of this research showed marked enhancement in bone osteogenesis within six months after surgery. This results contradict with Schmitz and Hollinger (2001), who showed doubt about the effect of use of PRF. ${ }^{(12)}$

The used technique in this study to extract the PRF was easy, rapid and simple to be done at the time of surgery. These results coincide with Weibrich et al., (2002), who showed that, there were no complications from the blood draw and PRF preparation. (13)

All cases of this study healed properly with no incidence of occurrence of graft infection nor discharge except only one case (case no. 7) who was treated properly by intravenous antibiotics and improved at late follow-up i.e. (after 3 and 6 month from operation). This result agreed with Marukawa et al., (2011), who reported that, PRF inhibit bacterial growth due to: primarily it's acidic PH (6.5 6.7), secondarily, PRF, concentrates the WBCS and platelet to cause bacterial inhibition by greater number of functionally variable leukocytes, thirdly, PRF causes rapid development of granulation tissue by enhancement of in-growth of capillaries. So, bringing in circulating macrophages and neutrophils. ${ }^{(14)}$

According to the results of this study, no graft migration or extrusion had occurred in any case through the follow up period. This result agree with Man et al., (2001) who reported occurrence of proper healing in all cases who was treated with autogenous graft with PRF, with no case of graft rejection had occurred. ${ }^{(15)}$

In the present study, no pain had been detected in all cases at the end of follow-up (i.e. after 6 months from surgery). This result agreed with Chandan et al., (2018), who reported that, pain persisted longer in patients who were treated with autgenous graft than in patients who was treated with the combined use of PRF and autogenous graft. ${ }^{(16)}$

According to this study, all patients gained uneventful closure of the alveolar arch and no oronasal fistula had been detected at the end of the follow-up (i.e. after 6 months). This result agreed with, Strayhon et al., (1999), who showed no oronasal fistula had occurred in all treated cases. ${ }^{(17)}$

The radiographic results of this study by coronal (CT) views revealed proper increase in the graft height postoperatively through the follow up periods. This in agreement with Behina et al., (2009), who reported proper increase in bone height in the reconstructed cleft defect during follow-up periods. ${ }^{(18)}$

During evaluating the bone density of the bone bridge using CT scan during the follow-up periods revealed increase in the bone density over the time, which can be explained by occurrence of continuous and early bone deposition. This result was consistent with the study of Marx et al., (1999), who reported occurrence of increase in bone mineral density in PRF supported grafts ranging from 1.6 to 2.2 times that of a non PRF supported graft. ${ }^{(19)}$ 


\section{CONCLUSION}

From the obtained results of this study we can conclude:

1. Obtaining bone graft from the anterior aspect of iliac crest is easy and safe method for harvesting adequate amount of autogenous bone graft.

2. PRF enhance bone formation in alveolar clefts when mixed with chips of autogenous iliac bone graft as it leads to occurrence of proper increase in bone height and bone density, in addition there is low infection rate and least postoperative complications

\section{REFERENCES}

1. Van AaLst JA, Kolappa KK, Sadove M. and Moc-Pssm CME, (2008): Non syndromic cleft palate. J.Plastic Reconstr Surg;121(1,suppl)1-14.

2. Theodor A. (1990): Digits in the mouth; a particular chapter in cleft lip and palate surgery .British Journal of Plastic surgery;43:724-727.

3. Seifeldin SA. (2016): Is alveolar cleft reconstruction still controversial ? (review of literature). Saudi Dent. J;28:311

4. Kawata T, Matsuki A and Kohno S.(2004): A new transplant bone for maxillary alveolar cleft. J.Exp. Amin.Sci, 43:19-28

5. La Rossa D., Buchmann S., Rothkopf DM., Mayro R. and Randall P.A. (1995): A comparison of iliac and cranial bone in secondary grafting of alveolar clefts. J.Plastic Reconst Surg;96:789-797.

6. Swan M. and Goodacre T. (2016): Morbidity of the iliac crest donor site following bone grafting of the cleft alveolus. British Journal of Oral and Maxillofacial Surgery;44:129-133.

7. Dohan DM, Choukroun J. and Diss A. (2006): Platelet rich fibrin (PRF): A second generation platelet concentrate. Part III: Leukocyte activation: A new feature for platelets concentrates. J. Oral Surg. Oral Med, Oral Path., oral Radiol, Endod.; 101:e51-e55.

8. Sunitha Raja V., and Munirathnam Naidu E.,(2018): Platelet rich fibrin : Evaluation of a second generation platelet concentrate. Indian Journal of Dental Research; 19:42-46.
9. Choukroun J. Diss A. and Simonpieri A. (2006): Platelet rich fibrin (PRF): A second generation platelet concentrate. Part IV: Clinical effects on tissue healing. J. Oral Surg. Oral Med, Oral Path., oral Radiol, Endod.; 101:e56-e60.

10. Hrtel L, Pogl C, and Henkel Ko (1999): Dental implants in alveolar cleft patients: A retrospective study. Journal Craniomaxilofacial Surg. ;27:354-359.

11. Dempf R., Teltzrow T., and Kramer FJ (2002): Alveolar bone grfting in patients with complete clefts: a comparative study between secondary and tertiary bone grafting. Cleft palate craniofacial journal, 39:18-23.

12. Schmitz JP. And Hollinger Jo. (2001): The biology of platelet rich fibrin. Journal of Oral and Maxillofacial Surgery, 59:1119-1125.

13. Weibrich G., Kleis WK and Hanfer G. (2002): Growth factors level in the platelet rich fibrin, produced by 2 different methods: cursan-type PRP kit versus PRP system. International Journal oral maxillofacial implant,17:184-189.

14. Marukawa E.,Oshina H., Lino G., Morita K. and Omura K (2011): Reduction of bone resorption by the application of platelet rich fibrin (PRF) in bone grafting of the alveolar cleft. Journal craniomaxillofacial surgery,39(4):278-283.

15. Man D., Plosker H. and Winland JE. (2001): The use of autologous platelet rich fibrin (Platelet gel) and autologous platelet poor plasma (fibrin glue) in cosmetic surgery. J Plastic Reconstructive Surgery surgery,107: 229-237.

16. Chandan Gupta, Divya Mehrotra, Shadab Mohammad, Vaibhav Khanna, Gulshan Kumar Singh, Geeta Singh, Arul A.L Chellappa and Deepak Passi (2018): Alveolar bone graft with platelet rich fibrin in cleft alveolus. Journal of oral biology and craniofacial research;3:3-8.

17. Strayhon $\mathrm{Cl}$, Garrett Js., Dunn RL, Benedict JJ and Somerman MJ. (1999): Growth factors regulate expression of osteoblast associated genes. Journal periodontology;70:1345-1354.

18. Behina H, Khajasteh A, Soleimani M., Tehrenchi A., Khoshzaban A., Keshel SH and Atashi R., (2009): Secondary repair of alveolar clefts using human mesenchymal stem cells. J. Oral Surg. Oral Med, Oral Path., oral Radiol, Endod.; 108:e1-e6.

19. Marx RE., Carlson ER., Eichstaedt RM., Schimmele SR., Strauss JE. And Georgef KR. (1999): Platelet rich fibrin. Growth factor enhancement for bone grafts. J. Oral Surg, Oral Med, Oral Path., oral Radiol, Endod.; 85:638-646. 